\title{
Effects of Herbicide against Yield Attributes and Yield in Transplanted Rice
}

\author{
Durgesh Jaiswal and D. K. Gupta* \\ IGKV, RMD College of Agri. and Res. Station, Ambikapur, India \\ *Corresponding author
}

\begin{tabular}{|l|}
\hline Ke y w o r d s \\
Herbicides, \\
Transplanted rice, \\
Yield and Yield \\
attributes
\end{tabular}

\section{A B S T R A C T}

A field experiment was conducted during the kharif season 2019 at Instructional-cumResearch Farm, Raj Mohini Devi College of Agriculture \& Research Station, Ambikapur (C.G.) to evaluate the "Effect of herbicide against complex weed flora in transplanted rice, under Northern hill zone of Chhattisgarh. The experiment was laid out in 10 treatments $\mathrm{T}_{1}$ - Pretilachlor + Pyrazosulfuron (Ready mix) $769 \mathrm{~g} / \mathrm{ha}$ as PE at 0-5 DAT, $\mathrm{T}_{2}$ - Pretilachlor + Pyrazosulfuron (Ready mix) $922 \mathrm{~g} / \mathrm{ha}$ as PE at 0-5 DAT, $\mathrm{T}_{3}-$ Pretilachlor + Pyrazosulfuron (Ready mix) $615 \mathrm{~g} / \mathrm{ha}$ as PE at 0-5 DAT, $\mathrm{T}_{4}-$ Bensulfuron - methyl + Pretilachlor 660 $\mathrm{g} / \mathrm{ha}$ as PE at 0-5 DAT, $\mathrm{T}_{5}$ - Pyrazosulfuron - ethyl $15 \mathrm{~g}$ a.i / ha as PE at 0-5 DAT, $\mathrm{T}_{6}$ Penoxsulam $35 \mathrm{~g}$ a.i / ha as a POE at 20-25 DAT, $\mathrm{T}_{7}-$ Bispyribac $-\mathrm{Na}-$ Salt $25 \mathrm{~g}$ a.i/ ha as a POE at 20-25 DAT, $\mathrm{T}_{8}-$ Metsulfuron + Chlorimuron $4 \mathrm{~g}$ a.i. / ha as a POE at 12-15 DAT, $\mathrm{T}_{9}$ - Mechanical Weeding at $20 \& 40$ DAT and $\mathrm{T}_{10}-$ Weedy Check in RBD with three replications. The data indicated that significant higher growth parameter, yield attributes, yield and net return were obtained in mechanical weeding at 20 and 40 DAT, which was at par with treatment $\mathrm{T}_{2}$ - Pretilachlor + Pyrazosulfuron (Ready mix) $922 \mathrm{~g} / \mathrm{ha}$ as PE at 0-5 DAT, $\mathrm{T}_{1}$ - Pretilachlor + Pyrazosulfuron (Ready mix) $769 \mathrm{~g} / \mathrm{ha}, \mathrm{T}_{3}$ Pretilachlor + Pyrazosulfuron (Ready mix) $615 \mathrm{~g} /$ ha and $\mathrm{T}_{4}-$ Bensulfuron - methyl + Pretilachlor $660 \mathrm{~g} / \mathrm{ha}$. The economics analysis of yield also specified that treatment $\left(\mathrm{T}_{9}\right)$ mechanical weeding at 20 and 40 DAT recorded maximum net return and B:C ratio and which was at par with $\mathrm{T}_{2}$ - Pretilachlor + Pyrazosulfuron (Ready mix) $922 \mathrm{~g} / \mathrm{ha}$ as PE at 05 DAT, $\mathrm{T}_{1}$ - Pretilachlor + Pyrazosulfuron (Ready mix) $769 \mathrm{~g} / \mathrm{ha}, \mathrm{T}_{3}-$ Pretilachlor + Pyrazosulfuron (Ready mix) $615 \mathrm{~g} / \mathrm{ha}$ and $\mathrm{T}_{4}-$ Bensulfuron - methyl + Pretilachlor 660 $\mathrm{g} /$ ha but significantly superior over rest of the weed management treatments.

\section{Introduction}

In India rice crop occupies 44.0 million ha, and production of 110.15 million tonnes with productivity 2.55 tonnes ha. The rice plays a very vital role in the national food security. In India, rice is grown under three major ecosystem: rainfed uplands (16\%), rainfed lowlands (39\%) and irrigated lands (45\%) with a productivity of $0.87,1.55$, and 2.24 tonnes ha respectively. In Chhattisgarh during Kharif season rice is mainly grown under rainfed ecosystem and it is completely dependent on monsoon. Rice occupies an area of 3.68 million ha of total 5.9 million ha cultivated area with productivity of 2.02 
tonnes ha. Chhattisgarh state contributes 5.26 per cent of the total rice production of the country. However, the production and productivity of rice per unit area is very low compared to other state like Punjab (38.09 q/ha), Haryana (31.12 q/ha) and West Bengal (27.31 q/ha) (Anonymous, 2017). Severe weed infestation is a major biotic constraint for rice production. Uncontrolled weeds cause $10-80 \%$ reduction in grain yield and sometimes result in complete failure of crop. Manual weeding is a common practice in the state but it is less efficient and highly cost, labour intensive and often not done on time owing to adverse soil and weather conditions. So use of suitable herbicides provides more efficient and cost - effective control of weeds.

\section{Materials and Methods}

The experiment was conducted at Instructional-cum-Research Farm, Rajmohini Devi College of Agriculture and Research Station, Ambikapur (C.G.) during kharif 2019. The soil of the experimental field was sandy clay in texture with low available nitrogen (241 kg ha ${ }^{-1}$ ), medium available phosphorous (12.7 $\left.\mathrm{kg} \mathrm{ha}^{-1}\right)$, high available potassium content $\left(429.70 \mathrm{~kg} \mathrm{ha}^{-1}\right)$.

An field experiment was performed in the basic of randomized block design with three replication for ten treatment including viz. Pretilachlor + Pyrazosulfuron (Ready- mix) @ $922 \mathrm{~g} / \mathrm{ha}$ at (0-5 DAT), Pretilachlor + Pyrazosulfuron (Ready- mix) @ 769 g/ha at (0-5 DAT), Pretilachlor + Pyrazosulfuron (Ready- mix) @ 615g/ha at (0-5 DAT), Bensulfuron - methyl + Pretilachlor @ 660 g/ha at (0-5 DAT), Pyrazosulfuron - ethyl 15 g a.i / ha at (0-5 DAT), Penoxsulam @ $35 \mathrm{~g}$ a.i/ha at 20-25 DAT, Bispyribac - Na - Salt @ $25 \mathrm{~g} \mathrm{a.i} / \mathrm{ha}$ at 20-25 DAT, Metsulfuron + Chlorimuron @ 4g a.i./ha at 12-15 DAT, Mechanical weeding (20 and 40 DAT) and weedy check. The rice variety IR-64 was used in the experiment. Rice was transplanted in August and harvested in November 2019. A uniform fertilizer dose of $120,60,40 \mathrm{~kg} \mathrm{~N}$, $\mathrm{P}_{2} \mathrm{O}_{5}$ and $\mathrm{K}_{2} \mathrm{O}$ ha $^{-1}$ was applied in the experimental field. All the recommended agronomic and plant protection measures were adopted to raise the crop. The data on weed density and dry weight were recorded at different growth stages of rice crop. These were subjected to square root transformation to normalize their distribution. Weed control efficiency (\%) was computed using the values of dry weight of weeds. Grain yield of rice along with other yield attributing characters were recorded at harvest.

\section{Results and Discussion}

\section{Effect on weeds}

The experimental field was infested with all three categories of weeds under seven families. The total number of major species were fourteen, out of which Echinochloa colona, Echinochloa crusgali, Cynodon dactylon, Leptochloa chinensis, Digitaria sanguinalis among grasses, Cyperus iria, Fimbristylis miliacea, Cyperus rotandus, Cyperus difformis among sedges and Eclipta alba, Commelina benghalensis, Eichhornia crassipes, Ludwigia parviflora, Ammania baccifera among broad leaf weed were present in the transplanted rice field.

All weed management practices brought significant variation in density and dry weight of weeds. Mechanical weeding at 20 and 40 DAT recorded the lowest number $(5.66,6.12$, and $5.53 \mathrm{no} / \mathrm{m}^{2}$ ) as well as dry weight of grasses, sedges, broad leaved weeds (2.58, 3.99 and $4.50 \mathrm{~g} / \mathrm{m}^{2}$ ) and highest weed control efficiency $(81.61,60.89$ and $61.43 \%$ ) at 30,60 and 90 DAT respectively. Which was at par with Pretilachlor + Pyrazosulfuron (Ready mix) $922 \mathrm{~g} / \mathrm{ha}$ as PE at 0-5 DAT fb Pretilachlor + Pyrazosulfuron (Ready mix) 
$769 \mathrm{~g}$ /ha as PE at 0-5 DAT, Pretilachlor + Pyrazosulfuron (Ready mix) $615 \mathrm{~g} / \mathrm{ha}$ as PE at 0-5 DAT and Bensulfuron - methyl + Pretilachlor $660 \mathrm{~g} / \mathrm{ha}$ as PE at 0-5 DAT. This result was similar to that of the experiment at findings of Yadav et al., (2007). The lower weed density with that treatment might be due to inherent ability of chemical to affect the cell division, cell growth and hampering the germination of weeds. Similar findings were reported by (Manhas et al.(2012), Kumar et al., (2014), Duary et al., (2015), Teja et al., (2015).

Table.1 Effect of herbicides against weed density and dry weight on transplanted rice

\begin{tabular}{|c|c|c|c|c|c|c|}
\hline \multirow[t]{2}{*}{ Treatments } & \multicolumn{3}{|c|}{ Total weed density $/ \mathrm{m}^{2}$} & \multicolumn{3}{|c|}{$\begin{array}{l}\text { Total weed dry } \\
\text { weight } / \mathrm{m}^{2}\end{array}$} \\
\hline & $\begin{array}{c}\text { 30 } \\
\text { DAT }\end{array}$ & $\begin{array}{c}\text { 60 } \\
\text { DAT }\end{array}$ & $\begin{array}{c}90 \\
\text { DAT }\end{array}$ & $\begin{array}{c}\text { 30 } \\
\text { DAT }\end{array}$ & $\begin{array}{c}\text { 60 } \\
\text { DAT }\end{array}$ & $\begin{array}{c}90 \\
\text { DAT }\end{array}$ \\
\hline $\begin{array}{l}\mathrm{T}_{1} \text { - Pretilachlor + Pyrazosulfuron } \\
\text { (Ready mix) } 769 \mathrm{~g} \text { a.i /hac. as PE } \\
\text { at 0-5 DAT }\end{array}$ & $\begin{array}{l}(8.58) \\
72.62\end{array}$ & $\begin{array}{l}(10.13) \\
101.76\end{array}$ & $\begin{array}{l}(8.22) \\
66.73\end{array}$ & $\begin{array}{l}(3.49) \\
11.21\end{array}$ & $\begin{array}{l}(4.86) \\
22.66\end{array}$ & $\begin{array}{l}(5.37) \\
27.88\end{array}$ \\
\hline $\begin{array}{l}\mathrm{T}_{2} \text { - Pretilachlor + Pyrazosulfuron } \\
\text { (Ready mix) } 922 \text { g a.i / hac. as PE } \\
\text { at 0-5 DAT }\end{array}$ & $\begin{array}{l}(8.05) \\
63.82\end{array}$ & $\begin{array}{l}(9.39) \\
87.25)\end{array}$ & $\begin{array}{c}(7.98) \\
62.7\end{array}$ & $\begin{array}{c}(3.22) \\
9.43\end{array}$ & $\begin{array}{l}(4.55) \\
19.77\end{array}$ & $\begin{array}{l}(5.15) \\
25.54\end{array}$ \\
\hline $\begin{array}{l}T_{3} \text { - Pretilachlor + Pyrazosulfuron } \\
\text { (Ready mix) } 615 \text { g a.i /hac. as PE } \\
\text { at 0-5 DAT }\end{array}$ & $\begin{array}{l}(9.24) \\
84.85\end{array}$ & $\begin{array}{l}(10.89) \\
117.69\end{array}$ & $\begin{array}{l}(9.36) \\
86.62\end{array}$ & $\begin{array}{l}(3.82) \\
13.66\end{array}$ & $\begin{array}{c}(5.01) \\
24.0\end{array}$ & $\begin{array}{l}(5.62) \\
30.66\end{array}$ \\
\hline $\begin{array}{l}\mathrm{T}_{4} \text { - Bensulfuron - methyl }+ \\
\text { Pretilachlor } 660 \mathrm{~g} \text { a.i / hac. as PE } \\
\text { at 0-5 DAT }\end{array}$ & $\begin{array}{l}(9.63) \\
91.85\end{array}$ & $\begin{array}{l}(11.57) \\
133.08\end{array}$ & $\begin{array}{l}(9.87) \\
96.53\end{array}$ & $\begin{array}{l}(3.87) \\
14.00\end{array}$ & $\begin{array}{c}(5.29) \\
27.0\end{array}$ & $\begin{array}{l}(5.68) \\
31.33\end{array}$ \\
\hline $\begin{array}{l}\mathrm{T}_{5} \text { - Pyrazosulfuron - ethyl } 15 \mathrm{~g} \text { a.i / } \\
\text { hac as PE at 0-5 DAT }\end{array}$ & $\begin{array}{l}(10.40) \\
107.21\end{array}$ & $\begin{array}{l}(11.91) \\
140.94\end{array}$ & $\begin{array}{c}(10.91) \\
118.2\end{array}$ & $\begin{array}{l}(4.08) \\
15.66\end{array}$ & $\begin{array}{l}(5.37) \\
27.88\end{array}$ & $\begin{array}{l}(6.51) \\
41.33\end{array}$ \\
\hline $\begin{array}{l}\mathrm{T}_{6}-\text { Penoxsulam } 35 \mathrm{~g} \text { a.i / hac. as a } \\
\text { POE at 20-25 DAT }\end{array}$ & $\begin{array}{l}(12.12) \\
146.01\end{array}$ & $\begin{array}{l}(13.32) \\
176.65\end{array}$ & $\begin{array}{l}(11.78) \\
137.84\end{array}$ & $\begin{array}{l}(4.88) \\
22.88\end{array}$ & $\begin{array}{l}(5.85) \\
33.32\end{array}$ & $\begin{array}{l}(6.67) \\
43.55\end{array}$ \\
\hline $\begin{array}{c}\mathrm{T}_{7}-\text { Bispyribac }-\mathrm{Na}-\text { Salt } 25 \mathrm{~g} \text { a.i/ } \\
\text { hac as a POE at 20-25 DAT }\end{array}$ & $\begin{array}{l}(11.57) \\
133.06\end{array}$ & $\begin{array}{l}(12.67) \\
159.58\end{array}$ & $\begin{array}{l}(10.60) \\
111.42\end{array}$ & $\begin{array}{l}(4.34) \\
17.88\end{array}$ & $\begin{array}{l}(5.79) \\
32.55\end{array}$ & $\begin{array}{l}(6.29) \\
38.66\end{array}$ \\
\hline $\begin{array}{c}\mathrm{T}_{8} \text { - Metsulfuron }+ \text { Chlorimuron } 4 \mathrm{~g} \\
\text { a.i/ hac. as a POE at 12-15 DAT }\end{array}$ & $\begin{array}{l}(10.95) \\
119.02\end{array}$ & $\begin{array}{l}(12.53) \\
156.17\end{array}$ & $\begin{array}{l}(11.39) \\
128.88\end{array}$ & $\begin{array}{l}(4.58) \\
19.99\end{array}$ & $\begin{array}{l}(5.86) \\
33.44\end{array}$ & $\begin{array}{l}(6.42) \\
40.32\end{array}$ \\
\hline $\begin{array}{l}\text { T9- Mechanical Weeding at } 20 \& 40 \\
\text { DAT }\end{array}$ & $\begin{array}{l}(5.66) \\
31.06\end{array}$ & $\begin{array}{l}(6.12) \\
36.57\end{array}$ & $\begin{array}{l}(5.53) \\
29.63\end{array}$ & $\begin{array}{c}(2.58) \\
5.66\end{array}$ & $\begin{array}{l}(3.99) \\
14.99\end{array}$ & $\begin{array}{l}(4.50) \\
19.32\end{array}$ \\
\hline $\mathbf{T}_{10}$ - Weedy Check & $\begin{array}{l}(15.19) \\
229.87\end{array}$ & $\begin{array}{l}(17.74) \\
313.75\end{array}$ & $\begin{array}{l}(14.81) \\
218.5\end{array}$ & $\begin{array}{l}(5.64) \\
30.85\end{array}$ & $\begin{array}{l}(6.19) \\
38.33\end{array}$ & $\begin{array}{l}(7.15) \\
50.10\end{array}$ \\
\hline $\mathrm{SEm} \pm$ & 1.27 & 1.70 & 1.36 & 0.34 & 0.56 & 0.39 \\
\hline CD (at 5\%) & 4.08 & 5.50 & 4.40 & 1.28 & 1.79 & 1.23 \\
\hline
\end{tabular}

*Figures in parenthesis indicates the transformed value. $\sqrt{\mathrm{X}+1}$ 
Table.2 Effect of herbicides against weed control efficiency and weed index on transplanted rice

\begin{tabular}{|c|c|c|c|c|}
\hline \multirow[t]{2}{*}{ Treatments } & \multicolumn{3}{|c|}{ Weed control efficiency (\%) } & \multirow{2}{*}{$\begin{array}{l}\text { Weed } \\
\text { Index }\end{array}$} \\
\hline & 30 DAT & 60 DAT & 90 DAT & \\
\hline $\begin{array}{l}\text { T }_{1} \text { - Pretilachlor + Pyrazosulfuron (Ready mix) } 769 \mathrm{~g} \\
\text { a.i /hac. as PE at 0-5 DAT }\end{array}$ & 63.66 & 40.88 & 44.35 & 2.55 \\
\hline $\begin{array}{l}\mathrm{T}_{2} \text { - Pretilachlor + Pyrazosulfuron (Ready mix) } 922 \mathrm{~g} \\
\text { a.i / hac. as PE at 0-5 DAT }\end{array}$ & 69.43 & 48.42 & 49.02 & 1.42 \\
\hline $\begin{array}{l}T_{3}-\text { Pretilachlor + Pyrazosulfuron (Ready mix) } 615 \mathrm{~g} \\
\text { a.i /hac. as PE at 0-5 DAT }\end{array}$ & 55.72 & 37.38 & 38.80 & 8.92 \\
\hline $\begin{array}{l}\mathrm{T}_{4} \text { - Bensulfuron - methyl + Pretilachlor } 660 \mathrm{~g} \text { a.i / } \\
\text { hac. as PE at 0-5 DAT }\end{array}$ & 54.61 & 29.55 & 37.46 & 9.18 \\
\hline $\begin{array}{l}T_{5} \text { - Pyrazosulfuron - ethyl } 15 \mathrm{~g} \text { a.i / hac as PE at 0-5 } \\
\text { DAT }\end{array}$ & 49.23 & 27.26 & 17.50 & 21.42 \\
\hline $\mathrm{T}_{6}$ - Penoxsulam $35 \mathrm{~g}$ a.i / hac. as a POE at 20-25 DAT & 25.83 & 13.07 & 13.07 & 29.08 \\
\hline $\begin{array}{l}\mathrm{T}_{7}-\text { Bispyribac }-\mathrm{Na}-\text { Salt } 25 \mathrm{~g} \text { a.i/ hac as a POE at } \\
\text { 20-25 DAT }\end{array}$ & 42.04 & 15.07 & 22.83 & 26.02 \\
\hline $\begin{array}{l}\mathrm{T}_{8} \text { - Metsulfuron + Chlorimuron } 4 \mathrm{~g} \text { a.i/ hac. as a POE } \\
\text { at 12-15 DAT }\end{array}$ & 35.2 & 12.75 & 19.52 & 23.46 \\
\hline$T_{9}$ - Mechanical Weeding at $20 \& 40$ DAT & 81.65 & 60.89 & 61.43 & -------- \\
\hline$T_{10}$ - Weedy Check & --- & --- & --- & 31.63 \\
\hline
\end{tabular}

Table.3 Effect of herbicide against yield attributes in transplanted rice

\begin{tabular}{|c|c|c|c|}
\hline Treatments & $\begin{array}{c}\text { Panicle } \\
\text { length }(\mathrm{cm})\end{array}$ & $\begin{array}{c}\text { Panicle } \\
\text { weight }(\mathrm{gm})\end{array}$ & $\begin{array}{l}\text { Grains per } \\
\text { panicle (no.) }\end{array}$ \\
\hline $\begin{array}{l}\mathrm{T}_{1} \text { - Pretilachlor + Pyrazosulfuron (Ready mix) } 769 \mathrm{~g} \\
\text { /ha as PE at 0-5 DAT }\end{array}$ & 18.33 & 2.4 & 99.33 \\
\hline $\begin{array}{l}\mathrm{T}_{2}-\text { Pretilachlor + Pyrazosulfuron (Ready mix) } 922 \mathrm{~g} \\
\text { / ha as PE at 0-5 DAT }\end{array}$ & 20 & 2.6 & 99.66 \\
\hline $\begin{array}{l}\mathrm{T}_{3} \text { - Pretilachlor + Pyrazosulfuron (Ready mix) } 615 \mathrm{~g} \\
\text { /ha as PE at 0-5 DAT }\end{array}$ & 18.33 & 2.33 & 98 \\
\hline $\begin{array}{l}\mathrm{T}_{4} \text { - Bensulfuron - methyl + Pretilachlor } 660 \mathrm{~g} / \mathrm{ha} \\
\text { as PE at 0-5 DAT }\end{array}$ & 17.66 & 2.32 & 91.66 \\
\hline $\begin{array}{l}\text { T }_{5}-\text { Pyrazosulfuron - ethyl } 15 \mathrm{~g} \text { a.i / ha as PE at 0-5 } \\
\text { DAT }\end{array}$ & 16.6 & 2.17 & 91.33 \\
\hline $\mathrm{T}_{6}$ - Penoxsulam $35 \mathrm{~g}$ a.i / ha as a POE at 20-25 DAT & 16.33 & 2.04 & 78 \\
\hline $\begin{array}{l}\text { T }_{7}-\text { Bispyribac }- \text { Na }- \text { Salt } 25 \mathrm{~g} \text { a.i/ ha as a POE at } \\
\text { 20-25 DAT }\end{array}$ & 16.4 & 2.04 & 82 \\
\hline $\begin{array}{l}\mathrm{T}_{8} \text { - Metsulfuron + Chlorimuron } 4 \mathrm{~g} \text { / ha as a POE } \\
\text { at 12-15 DAT }\end{array}$ & 16.5 & 2.12 & 84.33 \\
\hline$T_{9}$ - Mechanical Weeding at 20 \& 40 DAT & 20.66 & 2.66 & 100 \\
\hline$T_{10}$ - Weedy Check & 15.4 & 1.78 & 75.66 \\
\hline $\mathrm{SEm} \pm$ & 1.31 & 0.11 & 3.05 \\
\hline CD (at 5\%) & 4.1 & 0.35 & 9.32 \\
\hline
\end{tabular}


Table.4 Effect of herbicide against grain yield, straw yield, and harvest index in transplanted rice

\begin{tabular}{|c|c|c|c|}
\hline Treatments & $\begin{array}{l}\text { Grain yield } \\
\text { (q/ha) }\end{array}$ & $\begin{array}{l}\text { Straw yield } \\
\text { (q/ha) }\end{array}$ & $\begin{array}{c}\text { Harvest } \\
\text { index }(\%)\end{array}$ \\
\hline $\begin{array}{l}\mathrm{T}_{1}-\text { Pretilachlor + Pyrazosulfuron (Ready mix) } 769 \mathrm{~g} \\
\text { /ha as PE at 0-5 DAT }\end{array}$ & 63.66 & 79 & 44.62 \\
\hline $\begin{array}{l}\mathrm{T}_{2}-\text { Pretilachlor + Pyrazosulfuron (Ready mix) } 922 \mathrm{~g} \\
\text { / ha as PE at 0-5 DAT }\end{array}$ & 64.4 & 79.59 & 44.76 \\
\hline $\begin{array}{l}\mathrm{T}_{3}-\text { Pretilachlor + Pyrazosulfuron (Ready mix) } 615 \mathrm{~g} \\
\text { /ha as PE at 0-5 DAT }\end{array}$ & 59.5 & 78 & 43.26 \\
\hline $\begin{array}{l}\mathrm{T}_{4} \text { - Bensulfuron - methyl + Pretilachlor } 660 \mathrm{~g} / \mathrm{ha} \\
\text { as PE at 0-5 DAT }\end{array}$ & 59.33 & 77.83 & 43.25 \\
\hline $\begin{array}{l}\mathrm{T}_{5} \text { - Pyrazosulfuron - ethyl } 15 \mathrm{~g} \text { a.i / ha as PE at 0-5 } \\
\text { DAT }\end{array}$ & 51.33 & 72.5 & 41.35 \\
\hline $\mathrm{T}_{6}$ - Penoxsulam $35 \mathrm{~g}$ a.i / ha as a POE at 20-25 DAT & 46.33 & 67.66 & 40.65 \\
\hline $\begin{array}{l}\mathrm{T}_{7}-\text { Bispyribac }-\mathrm{Na}-\text { Salt } 25 \mathrm{~g} \text { a.i/ ha as a POE at } \\
\text { 20-25 DAT }\end{array}$ & 48.33 & 71 & 40.95 \\
\hline $\begin{array}{l}\mathrm{T}_{8}-\text { Metsulfuron }+ \text { Chlorimuron } 4 \mathrm{~g} / \text { ha as a POE } \\
\text { at 12-15 DAT }\end{array}$ & 50 & 72 & 41 \\
\hline$T_{9}$ - Mechanical Weeding at $20 \& 40$ DAT & 65.33 & 79.66 & 45 \\
\hline$T_{10}$ - Weedy Check & 44.66 & 64.5 & 40.6 \\
\hline $\mathrm{SEm} \pm$ & 2.05 & 2.33 & 0.74 \\
\hline CD (at 5\%) & 6.2 & 7.25 & 2.31 \\
\hline
\end{tabular}

Table.5 Effect of herbicide against gross, return, net return and B: C ratio in transplanted rice

\begin{tabular}{|c|c|c|c|}
\hline Treatments & $\begin{array}{l}\text { Gross } \\
\text { return } \\
\text { (Rs/ha) }\end{array}$ & $\begin{array}{l}\text { Net return } \\
\text { (Rs/ha) }\end{array}$ & B:C ratio \\
\hline $\begin{array}{l}\mathrm{T}_{1} \text { - Pretilachlor + Pyrazosulfuron (Ready mix) } 769 \mathrm{~g} / \mathrm{ha} \text { as } \\
\text { PE at 0-5 DAT }\end{array}$ & 131343 & 88942 & 2.1 \\
\hline $\begin{array}{l}T_{2}-\text { Pretilachlor + Pyrazosulfuron (Ready mix) } 922 \mathrm{~g} / \text { ha as } \\
\text { PE at 0-5 DAT }\end{array}$ & 132786 & 89635 & 2.08 \\
\hline $\begin{array}{l}\mathrm{T}_{3}-\text { Pretilachlor + Pyrazosulfuron (Ready mix) } 615 \mathrm{~g} / \mathrm{ha} \text { as } \\
\text { PE at 0-5 DAT }\end{array}$ & 123592 & 81941 & 1.97 \\
\hline $\begin{array}{l}\mathrm{T}_{4} \text { - Bensulfuron - methyl + Pretilachlor } 660 \mathrm{~g} / \mathrm{ha} \text { as PE at } \\
\text { 0-5 DAT }\end{array}$ & 123249 & 81773 & 1.97 \\
\hline $\mathrm{T}_{5}$ - Pyrazosulfuron - ethyl $15 \mathrm{~g}$ a.i / ha as PE at 0-5 DAT & 107724 & 68550 & 1.75 \\
\hline $\mathrm{T}_{6}$ - Penoxsulam $35 \mathrm{~g}$ a.i / ha as a POE at 20-25 DAT & 97621 & 56050 & 1.35 \\
\hline $\begin{array}{l}\mathrm{T}_{7}-\text { Bispyribac - Na - Salt } 25 \mathrm{~g} \text { a.i/ ha as a POE at } 20-25 \\
\text { DAT }\end{array}$ & 101920 & 61020 & 1.49 \\
\hline $\begin{array}{l}\text { T }_{8} \text { - Metsulfuron + Chlorimuron } 4 \mathrm{~g} \text { / ha as a POE at 12-15 } \\
\text { DAT }\end{array}$ & 105150 & 65949 & 1.68 \\
\hline $\mathrm{T}_{9}$ - Mechanical Weeding at $20 \& 40 \mathrm{DAT}$ & 134506 & 91230 & 2.11 \\
\hline$T_{10}$ - Weedy Check & 93960 & 56965 & 1.47 \\
\hline SEm \pm & 3675 & 3474 & 0.08 \\
\hline CD (at 5\%) & 11425 & 10403 & 0.24 \\
\hline
\end{tabular}




\section{Effect on crop}

Mechanical weeding at 20 and 40 DAT registered the significantly highest value of plant fresh and dry weight, yield attributes viz., panicle length $(20.66 \mathrm{~cm})$, panicle weight (2.66 gm), grains panicle- ${ }^{1}$ (100), highest grain $(65.33 \mathrm{q} / \mathrm{ha})$, straw yield $(79.66 \mathrm{q} / \mathrm{ha})$ and harvest index (45\%), net returns (9130 $\mathrm{Rs} / \mathrm{ha})$ and B:C (2.11) ratio and at par with Pretilachlor + Pyrazosulfuron (Ready mix) $922 \mathrm{~g} / \mathrm{ha}$ as PE at 0-5 DAT fb - Pretilachlor + Pyrazosulfuron (Ready mix) $769 \mathrm{~g} / \mathrm{ha}$ as $\mathrm{PE}$ at 0-5 DAT, Pretilachlor + Pyrazosulfuron (Ready mix) $615 \mathrm{~g} / \mathrm{ha}$ as PE at 0-5 DAT, Bensulfuron - methyl + Pretilachlor $660 \mathrm{~g} / \mathrm{ha}$ as PE at 0-5 DAT. This might be due to increased production and translocation of photosynthesis to yield attributes to adequate availability of growth resources and less competition during the crucial phase of crop offered by weeds. These result are in conformity with Kumar et al., (2014), Duary et al., (2015), Teja et al., (2015)

Mechanical weeding 20 and 40 DAT was found effective to control weeds in transplanted rice on the basis of vegetation analysis. However, based on economic analysis, mechanical weeding 20 and 40 DAT could be adjudged as the best treatment for effective and economic weed management and obtained higher yield.

\section{References}

Anonymous. (2017). Directorate of Economics and Statistics, Department of Agriculture and Cooperation, Ministry of Agriculture, Government of India.

Duary, B., Teja, K.C. and Soren, U. 2015. Management of composite weed flora of transplanted rice by herbicide. Indian Journal of Weed Science, 47(4): 349352.

Kumar, S., Rana, S.S., Navell, C. and Ramesh 2013. Mixed weed flora management by bispyribac-sodium in transplanted rice. Indian Journal of Weed Science 45(3): 151-155.

Manhas, S.S., Singh, G., Singh, D. and Khajuria, V. 2012. Effect of tank-mixed herbicides on weeds and transplanted rice (Oryza sativa L.). Annals of Agricultural Research, 33(1\&2): 25-31.

Teja, K.C., Duary, B. and Dash, S. 2016. Sole and combined application of herbicides on composite weed flora of transplanted rice. Indian Journal of Weed Science 48(3): 254-258.

\section{How to cite this article:}

Durgesh Jaiswal and Gupta, D. K. 2020. Effects of Herbicide against Yield Attributes and Yield in Transplanted Rice. Int.J.Curr.Microbiol.App.Sci. 9(10): 2038-2043.

doi: https://doi.org/10.20546/ijcmas.2020.910.248 